\title{
Clinical Correlation between Placido, Scheimpflug and LED Color Reflection Topographies in Imaging of a Scarred Cornea
}

\author{
Anastasios John Kanellopoulos ${ }^{a, b} \quad$ George Asimellis ${ }^{a}$ \\ ${ }^{a}$ Laservision.gr Eye Institute, Athens, Greece; ${ }^{b}$ New York University Medical School, \\ New York, N.Y., USA
}

\section{Key Words}

LED Cassini · Herpetic keratitis · Point-source topography · Differential topography · Color-coded topography · Pentacam HR · Corneal topography · Scheimpflug imaging · Irregular corneal astigmatism

\begin{abstract}
This case report aims to evaluate safety, efficacy and feasibility of anterior surface imaging by a novel point-source reflection topographer, in comparison to four other corneal imaging modalities. A 17-year-old female patient, clinically diagnosed with chronic herpetic keratitis in her left eye was imaged by a novel multicolored-spot reflection topography system. We comparatively investigated elevation and curvature maps between the novel topographer and established Placido disk topography and Scheimpflug tomography systems. Pachymetry maps were compared between the Scheimpflug system and anterior-segment optical coherence tomography system. The Placido system failed to properly register the abnormal anterior surface due to incomplete mire registration, while the Scheimpflug topometry device imaged the anterior surface properly, but not the posterior (due to media opacity), and thus pachymetry was highly irregular and erroneous in this case. Imaging of corneas infected with herpes simplex virus keratitis has been rare; we have not identified any such documentation in the peer review literature in the last 10 years. This novel multicolored-spot reflection topography imaging may offer successful corneal imaging in cases where established clinical topography systems may fail to produce accurate reconstruction of the corneal shape. This is an important case demonstrating exceptional clinical feasibility in such rare cases offered by a newly introduced technology in ophthalmic imaging.


Kanellopoulos and Asimellis: Clinical Correlation between Placido, Scheimpflug and LED Color Reflection Topographies in Imaging of a Scarred Cornea

\section{Case Presentation}

We present the case of a 17-year-old female diagnosed with chronic herpes simplex virus (HSV) keratitis [1] on her left eye, affected at a very young age (20 months of age). Informed consent was obtained from the subject at the time of the first clinical visit. This study adhered to the tenets of the Declaration of Helsinki and was approved by the Ethics Committee of our Institution.

This young patient has had multiple reacts of disciform (stromal) keratitis. We have treated her aggressively with topical corticosteroid therapy and oral antibiotics over the last year. We decided to assess the cornea irregularity caused by the inflammatory stromal response since this case was stable over the last 6 months.

The nonaffected right eye had uncorrected distance visual acuity 20/20 and manifest refraction $-0.50-0.25 \times 80^{\circ}$. The affected left eye had uncorrected distance visual acuity $20 / 40$, manifest refraction $+2.00-7.50 \times 170^{\circ}$, and best-spectacle corrected distance visual acuity with this prescription of $20 / 32$.

We have recently reported [2] the clinical results of a novel topography clinical system based on anterior corneal surface imaging by reflection of color-coded light-emitting diode (LED) spots array. Topography systems with color-coded point-source reflection forward ray-tracing have been proposed [3] as alternatives to Placido ring imaging [4], enabling, among other, the proper imaging of radial, rather than only contour topographic changes [5]. The VU topographer (Vrije Universiteit Medical Center, Amsterdam, The Netherlands) [3] introduced a color-coded square chess-pattern array projected on the cornea instead of the Placido rings [6]. The surface reconstruction algorithm employed data from the pattern crossing points to eliminate source-image mismatch. In principle, this color-coded topographer was found to be efficient in reconstructing the nonrotationally symmetric anterior corneal surface [7].

The Cassini (i-Optics BV, The Hague, The Netherlands) topography system is a novel topographer employing multispot (up to 700), multicolor (red, yellow and green) LED tear film-reflection-imaging, following the steps of the VU topographer. The difference is that instead of a limited number of color-coded squares, there are over 600 LED spots on the radial and contour arrangement imaged on the cornea. Image processing algorithm locates feature points in the reflection image and accounts for smearing and deformation in irregular corneas. Every three spots identified by the software are processed by ray tracing, and the relevant 'local' elevation is defined. This process is theoretically unbiased by media opacity and differs from the center to periphery dependence of data processing that occurs with Placido-disk topographers. The system has been recently introduced [8] and has received US Food and Drug Administration (FDA) approval for clinical use in corneal topography.

Due to the novelty, clinical validation of such systems and their clinical implications has yet to be investigated. The scope of this paper is to examine the clinical feasibility of a newly introduced point-source color-coded corneal topography in a case of keratitis imaging.

\section{Investigations}

Four anterior segment imaging modalities were studied in this work. Placido topography was provided by the Alcon/WaveLight Allegro Topolyzer (Alcon Surgical, Fort Worth, Tex., USA). The Topolyzer is a wide-cone corneal topographic Placido system with 22 concentric rings for the detection of up to 22,000 elevation points. Scheimpflug topometry 
Kanellopoulos and Asimellis: Clinical Correlation between Placido, Scheimpflug and LED Color Reflection Topographies in Imaging of a Scarred Cornea

was provided by the WaveLight Oculyzer II (Alcon Surgical), a Pentacam HR (highresolution) camera providing corneal pachymetry and topometry maps [9]. Anteriorsegment optical coherence tomography (AS-OCT) was employed for cornea cross-section, corneal pachymetry, and epithelial thickness maps [10]. The Fourier-domain AS-OCT system RTVue-100 (Optovue Inc., Fremont, Calif., USA), running on analysis and report software version A6 $(9,0,27)$ was employed for this purpose. Finally, the Cassini (running on software version 1.3, updated December 2013) was employed to provide anterior surface imaging. The system produces anterior elevation, tangential and axial curvature, and refractive power 3-dimensional maps covering the 7.5-mm diameter area. The report provides keratometry data (steep and flat SimK, simulated astigmatism), and computes topographic and keratoconus indices. In addition, aberrations report, according to the Zernike nomenclature, is provided. Four consecutive scans in each case were obtained by all systems.

Fig. 1 presents an OCT high-resolution meridian scan of the affected eye, and the corresponding Scheimpflug imaging raw scan. We observed the intracorneal scar, appearing as a stromal opacity associated with the residual stromal scarring, located in the near inferior nasal area. Fig. 2 presents a Placido ring imaging and the axial curvature map obtained with the Topolyzer. We observed the highly distorted ring pattern inferior nasally. It is evident that the system was unsuccessful to register proper information, as the axial curvature map was poorly calculated.

\section{Differential Diagnosis}

Fig. 3 presents corneal pachymetry derived from Scheimpflug imaging ( $9 \mathrm{~mm}$ diameter) and the corresponding OCT-derived ( $6 \mathrm{~mm}$ diameter) corneal and epithelial thickness maps.

Local corneal thinnest and epithelial hyperplasia are also identified in the same affected area. The imaging depicts successfully the corneal opacity, and produces a nearly complete axial curvature map. As it can be noticed in fig. 3a, the posterior surface is poorly identified at the lesion, as expected due to the corneal opacity. Thinnest pachymetry was reported by the Scheimpflug data at $380 \mu \mathrm{m}$, while by OCT at $480 \mu \mathrm{m}$. This case, with substantial opacity, alters the 'normal' densitometry interpolation of the algorithm. Fig. 4 presents comparatively data from the imaging on the affected eye indicating impressive agreement of the anterior surface elevation and curvature maps between the Cassini topographer and the Scheimpflug device. Scheimpflug imaging reports a bump (about $50 \mathrm{D}$ ) on the superior side of the map that is not present in the Color LED topography map. This partial agreement may be attributed to incomplete imaging by either device over the specific area or to imaging interpolation difficulties over the scarred region.

One-year of follow-up has been recommended to validate the anterior-segment imaging findings. Ocular scatter and cornea densitometry measurements may further establish the scar morbidity on visual performance of the patient.

\section{Discussion}

HSV keratitis and its sequelae is the most frequent cause of corneal blindness in the United States and the most common source of infectious blindness in the Western world [11, 12]. Subsequent corneal scarring, which may have severe impact on the anterior surface and tear film regularity, as well as stromal density, as indicated by the OCT imaging, renders Placido and Scheimpflug imaging in this case challenging. 
Kanellopoulos and Asimellis: Clinical Correlation between Placido, Scheimpflug and LED Color Reflection Topographies in Imaging of a Scarred Cornea

In this work, we examined the clinical feasibility of a newly introduced multicoloredspot reflection corneal topographer in a case of HSV keratitis in comparison to established methods of clinical imaging, namely OCT, Placido and Scheimpflug imaging.

Thinnest cornea pachymetry with Scheimpflug imaging (measured $380 \mu \mathrm{m}$ ) was in large disparity with the OCT thinnest pachymetry $(480 \mu \mathrm{m})$. We believe that this is a disadvantage of the Scheimpflug imaging principle, which assumes clear cornea interpolation of the posterior surface.

When comparing the Cassini to the Placido system, this case suggests that the increased sensitivity, which includes both radial and contour differences based on spot imaging, enabled proper imaging of this highly irregular cornea. Particularly considering the extent of the abnormality present in this case, and the fact that significant distortion was also located at the center, the Placido device was not successful in imaging this case. The Scheimpflug imaging was successful in imaging the anterior surface, but not the posterior.

The clinical relevance lies in cases where cornea curvature accuracy is needed, such as IOL power calculations.

\section{Conclusions}

This case report, based on LED color reflection topography imaging in comparison to Scheimpflug and Placido imaging, verifies the clinical applicability of the new technology in the imaging of disturbed and highly asymmetric cornea.

The facility and comparable results offered by the Cassini, in comparison to established Placido and Scheimpflug imaging, as well as the possibility of increased predictability that may be offered by the novel corneal imaging in highly irregular corneas, may hold promise for wider clinical application, such as screening of corneas either highly asymmetric and/or with highly coinciding opacities.

The potential higher specificity in the central cornea that seems to be unaffected by cornea opacities may offer a differential advantage in relation to other contemporary cornea imaging devices. Additionally, concerning highly distorted corneas, the every-3-spots-ray tracing calculation used with the Cassini may offer higher predictability in correlation to Placido-derived topography were a central irregularity may bias axially the relevant section of the map.

\section{References}

1 Kaye S, Choudhary A: Herpes simplex keratitis. Prog Retin Eye Res 2006;25:355-380.

2 Kanellopoulos AJ, Asimellis G: Forme Fruste Keratoconus Imaging and Validation via Novel Multi-spot Reflection Topography. Case Rep Ophthalmol 2013;4:199-209.

-3 Vos FM, Groen FCA, van Stokkum IHM, Spoelder HJW, van der Heijde GL: A new instrument to measure the shape of the cornea based on pseudorandom color coding. IEEE Trans Instrum Meas 1997;46:794-797.

4 Snellenburg JJ, Braaf B, Hermans EA, van der Heijde RG, Sicam VA: Forward ray tracing for image projection prediction and surface reconstruction in the evaluation of corneal topography systems. Opt Express 2010;18:19324-19338.

5 Rand RH, Howland HC, Applegate RA: Mathematical model of a Placido disk keratometer and its implications for recovery of corneal topography. Optom Vis Sci 1997;74:926-930.

-6 Sicam VA, Snellenburg JJ, van der Heijde RG, van Stokkum IH: Pseudo forward ray-tracing: a new method for surface validation in cornea topography. Optom Vis Sci 2007;84:915-923.

-7 Sicam VA, van der Heijde RG: Topographer reconstruction of the nonrotation-symmetric anterior corneal surface features. Optom Vis Sci 2006;83:910-918.

8 Weikert MP, Koch DD, Wang D: Evaluation of Corneal Topography Based on Color LED Technology, ASCRS Symposium and Congress 2013, San Francisco, USA. 
Case Reports in

Ophthalmology

\begin{tabular}{l|l}
\hline Case Rep Ophthalmol 2014;5:311-317 \\
\hline DOI: 10.1159/000365962 & $\begin{array}{l}\text { ○ 2014 S. Karger AG, Basel } \\
\text { www.karger.com/cop }\end{array}$ \\
\hline
\end{tabular}

Kanellopoulos and Asimellis: Clinical Correlation between Placido, Scheimpflug and LED Color Reflection Topographies in Imaging of a Scarred Cornea

-9 Kanellopoulos AJ, Asimellis G: Comparison of Placido disc and Scheimpflug image-derived topographyguided excimer laser surface normalization combined with higher fluence CXL: the Athens Protocol, in progressive keratoconus. Clin Ophthalmol 2013;7:1385-1396.

-10 Kanellopoulos AJ, Asimellis G: In vivo three-dimensional epithelial imaging of corneal epithelium in normal eyes by anterior segment optical coherence tomography: a clinical reference study. Cornea 2013;32:14931498.

11 Wilhelmus KR: Diagnosis and management of herpes simplex stromal keratitis. Cornea 1987;6:286-291.

$\$ 12$ Liesegang TJ, Melton LJ 3rd, Daly PJ, et al: Epidemiology of ocular herpes simplex. Incidence in Rochester, Minn, 1950 through 1982. Arch Ophthalmol 1989;107:1155-1159.

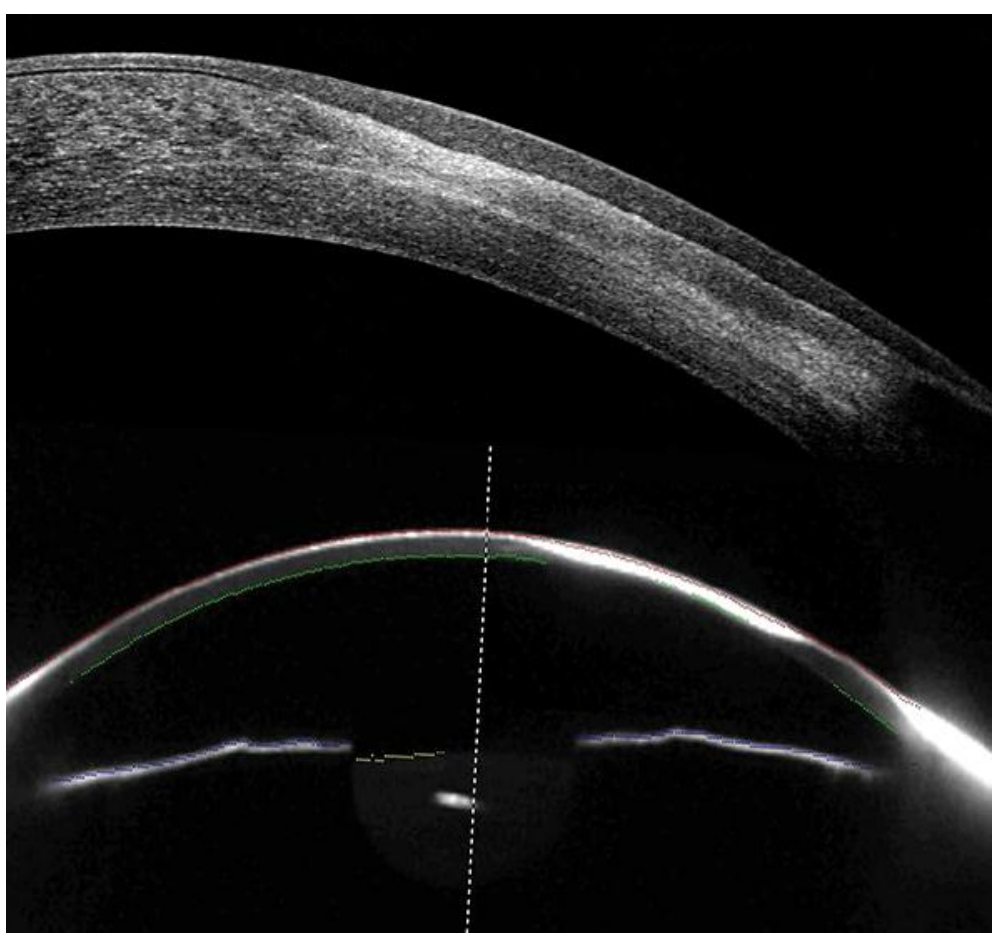

Fig. 1. Top: OCT high-resolution meridian $\left(80^{\circ}\right)$ scan of the affected HSV eye. Bottom: corresponding Scheimpflug imaging scan image. 
Case Reports in

Ophthalmology

\begin{tabular}{l|l}
\hline Case Rep Ophthalmol 2014;5:311-317 \\
\hline DOI: 10.1159/000365962 & $\begin{array}{l}\text { ○ 2014 S. Karger AG, Basel } \\
\text { www.karger.com/cop }\end{array}$ \\
\hline
\end{tabular}

Kanellopoulos and Asimellis: Clinical Correlation between Placido, Scheimpflug and LED Color Reflection Topographies in Imaging of a Scarred Cornea

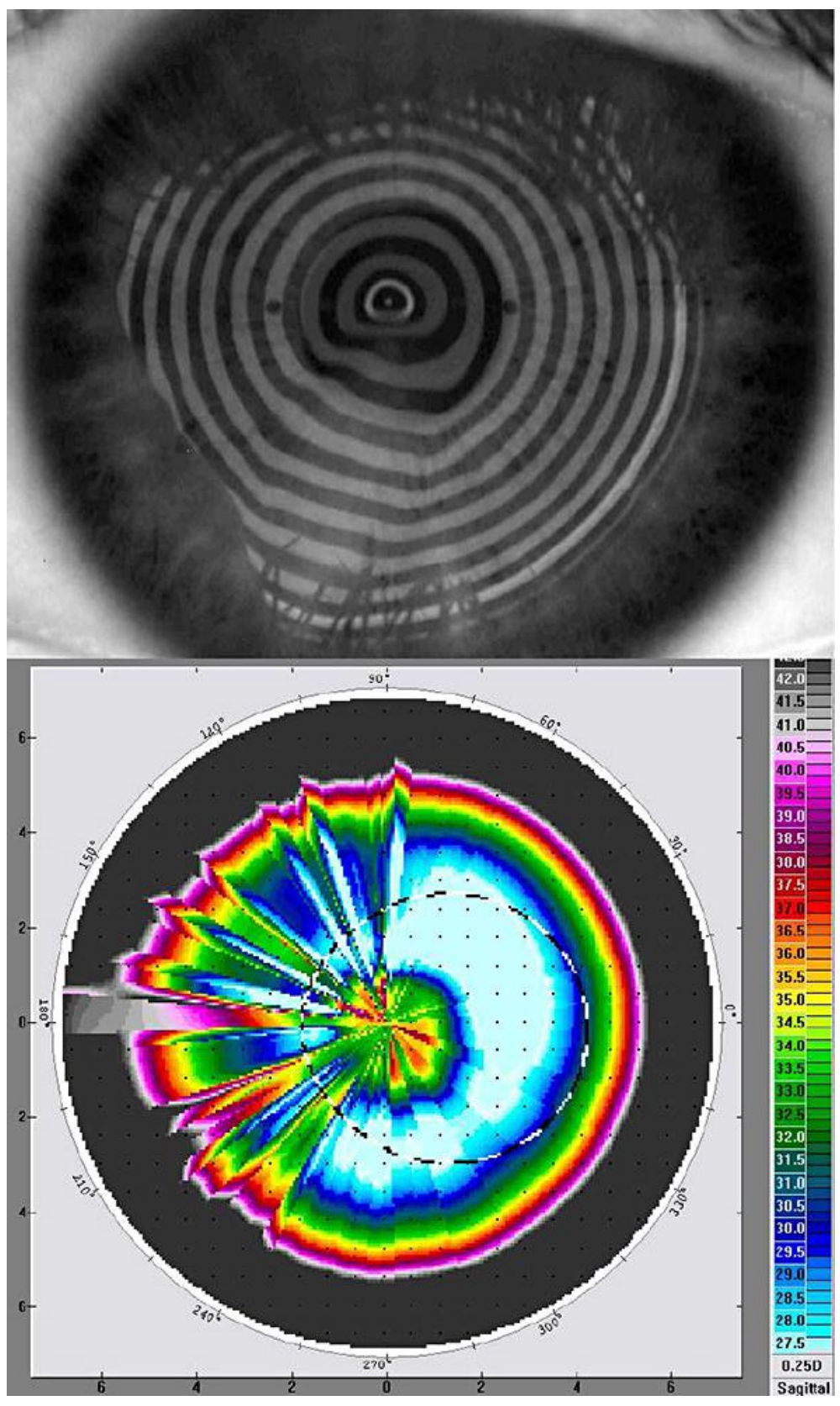

Fig. 2. Top: Placido ring imaging on the affected eye. Bottom: axial curvature map computed by the above pattern. 


\section{Case Reports in \\ Ophthalmology}

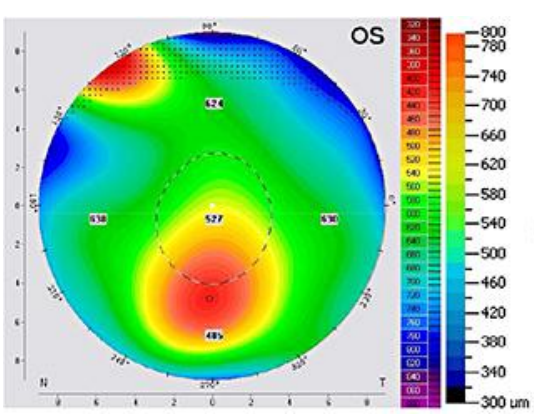

Case Rep Ophthalmol 2014;5:311-317

DOI: $10.1159 / 000365962$
C 2014 S. Karger AG, Basel

www.karger.com/cop

Kanellopoulos and Asimellis: Clinical Correlation between Placido, Scheimpflug and LED Color Reflection Topographies in Imaging of a Scarred Cornea

Fig. 3. Corneal pachymetry maps. Left: Scheimpflug imaging-derived corneal pachymetry $(9 \mathrm{~mm}$ diameter). Right: OCT-derived corneal and epithelial thickness pachymetry maps ( $6 \mathrm{~mm}$ diameter) of the same eye.
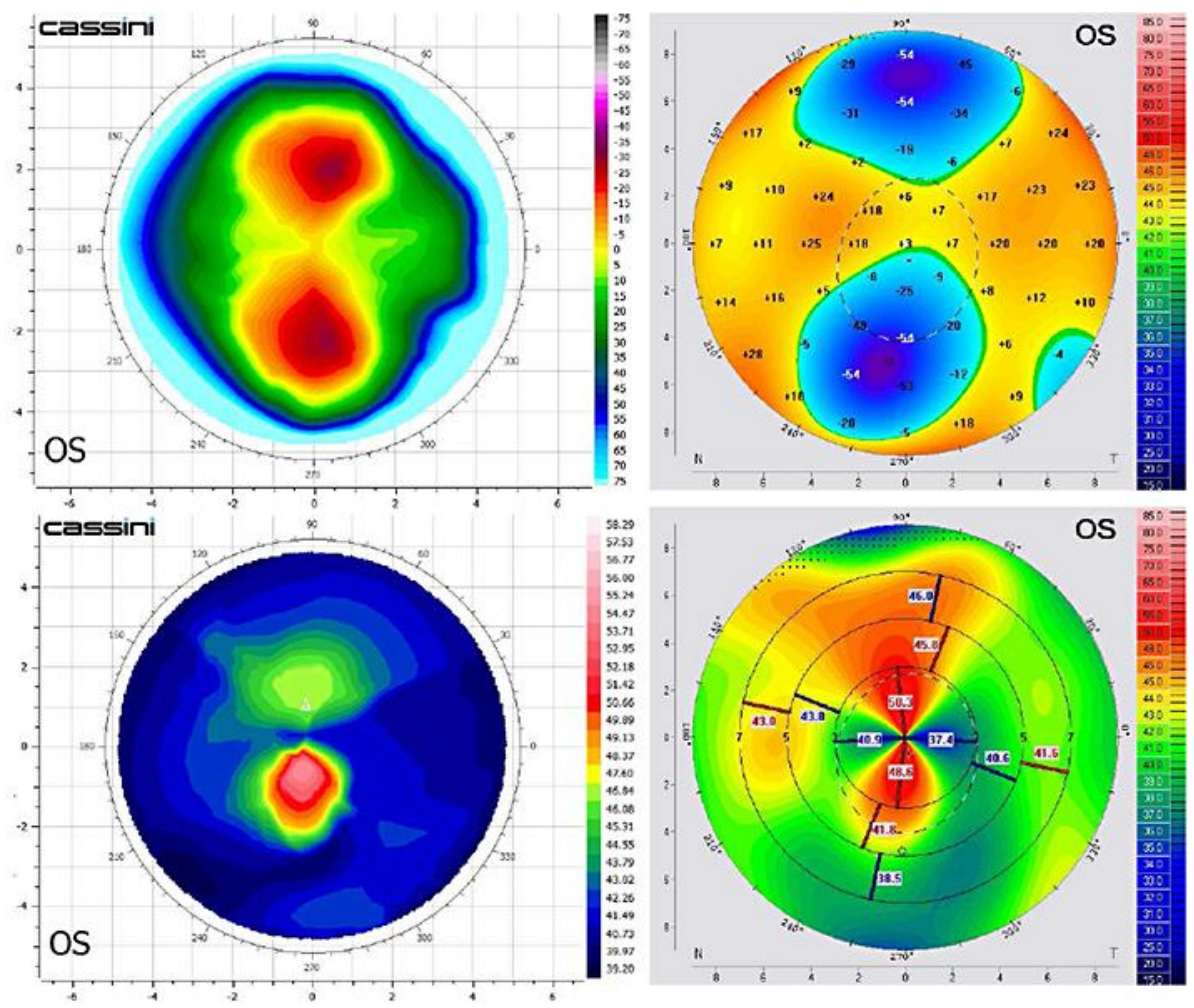

Fig. 4. Left: LED spot reflection topography-derived elevation (top left) and axial (bottom left) curvature maps. Right: the corresponding maps derived from Scheimpflug imaging. 